\title{
SYMMETRIC AND NON-SYMMETRIC DISCONTINUOUS GALERKIN METHODS STABILIZED USING BUBBLE ENRICHMENT
}

\author{
ERIK BURMAN AND BENJAMIN STAMM
}

\begin{abstract}
In this Note we prove that in two and three space dimensions, the symmetric and non-symmetric discontinuous Galerkin method for second order elliptic problems is stable when using piecewise linear elements enriched with quadratic bubbles without any penalization of the interelement jumps. The method yields optimal convergence rates in both the broken energy norm and, in the symmetric case, the $L^{2}$-norm. Moreover the method can be written in conservative form with fluxes independent of any stabilization parameter. To cite this article: E. Burman and B. Stamm, C. R. Acad. Sci. Paris, vol. 346, num. 1-2, 2008, p. 103-106.
\end{abstract}

\section{INTRODUCTION}

The Discontinuous Galerkin (DG) method is a classical technique to approximate elliptic and hyperbolic PDE's. For elliptic PDE's, two of the most popular methods are the Symmetric Interior Penalty (SIP) method introduced by Baker [3] and Arnold [1] and the non-symmetric DG method introduced by Oden, Babuška and Baumann [9]. One attractive feature of the latter method is that, because of the absence of penalty terms, it can be written in conservative form with fluxes that are independent of numerical parameters. Moreover, the non-symmetric DG method is proven to yield optimal convergence estimates in the broken energy norm in one $[7]$ and two space dimensions $[8,10]$ for polynomial orders $p \geq 2$, while for $p=1$, penalty terms must be introduced to grant stability and optimal convergence rates in the broken energy norm, but the conservative fluxes then depend on the penalty parameter.

Working with the SIP method instead of the non-symmetric DG method presents the twofold advantage of dealing with symmetric linear systems and of ensuring optimal convergence rates also in the $L^{2}$-norm. The difficulty with the SIP method is that stability usually relies on the use of penalty parameters that will subsequently enter the expression of the conservative fluxes.

The purpose of this Note is to fill the gap between symmetric and non-symmetric DG methods in the special case of piecewise linear approximation enriched with quadratic bubbles. We prove that both the non-symmetric and the symmetric DG method without any penalty leads to optimal convergence rates in the broken energy norm and in the $L^{2}$-norm (only for the symmetric version) in two and three space dimensions. A similar analysis for $p \geq 2$ was proposed in the one-dimensional case in [5] for the symmetric version. In the non-symmetric case, Brezzi and Marini,

This project received financial support from the Swiss National Science Foundation under grant $200021-113304$. 
recently proposed an analysis, also using bubble-stabilization [4]. We also refer to [2] for an overview of stabilization techniques for DG-methods. In this note proofs are only sketched and we refer to [6] for full detail.

\section{Model PROBlem AND METHOD FORMULATiON}

Let $\Omega$ be a convex polygon (polyhedron in three space dimensions) in $\mathbb{R}^{d}, d=2,3$, with outer normal $n$. Let $\mathcal{K}$ be a subdivision of $\Omega \subset \mathbb{R}^{d}$ into non-overlapping $d$ simplices $\kappa$. We consider the following elliptic problem with homogenous Dirichlet boundary conditions:

Find $u: \Omega \rightarrow \mathbb{R}$ such that

$$
\left\{\begin{aligned}
-\Delta u & =f, \text { in } \Omega \\
u & =0, \text { on } \partial \Omega .
\end{aligned}\right.
$$

Assume that $\mathcal{K}$ is shape-regular, does not contain any hanging node and covers $\bar{\Omega}$ exactly. Let $\mathcal{F}_{i}$ denote the set of interior faces $((d-1)$-manifolds $)$ of the mesh, i.e. the set of faces that are not included in the boundary $\partial \Omega$. The set $\mathcal{F}_{e}$ denotes the faces that are included in $\partial \Omega$ and define $\mathcal{F}=\mathcal{F}_{i} \cup \mathcal{F}_{e}$. For an element $\kappa \in \mathcal{K}$ or a face $F \in \mathcal{F}$ let $h_{\kappa}$ resp. $h_{F}$ denotes its diameter. Set $h=\max _{\kappa \in \mathcal{K}} h_{\kappa}$ and let $\tilde{h}$ and $\tilde{h}_{\mathcal{F}}$ be two functions such that $\left.\tilde{h}\right|_{\kappa}=h_{\kappa}$ and $\left.\tilde{h}_{\mathcal{F}}\right|_{F}=h_{F}$.

For a subset $R \subset \Omega$ or $R \subset \mathcal{F},(\cdot, \cdot)_{R}$ denotes the $L^{2}(R)$-scalar product, $\|\cdot\|_{R}=$ $(\cdot, \cdot)_{R}^{1 / 2}$ the corresponding norm, and $\|\cdot\|_{s, R}$ the $H^{s}(R)$-norm. The element-wise counterparts will be distinguished using the discrete partition as subscript, for example $(\cdot, \cdot)_{\mathcal{K}}=\sum_{\kappa \in \mathcal{K}}(\cdot, \cdot)_{K}$. For $s \geq 1$, let $H^{s}(\mathcal{K})$ be the space of piecewise Sobolev $H^{s}$-functions and denote its norm by $\|\cdot\|_{s, \mathcal{K}}$.

For $v \in H^{1}(\mathcal{K}), \tau \in\left[H^{1}(\mathcal{K})\right]^{d}$ and an interior face $F=\kappa_{1} \cap \kappa_{2} \in \mathcal{F}_{i}$, where $\kappa_{1}$ and $\kappa_{2}$ are two distinct elements of $\mathcal{K}$ with respective outer normals $n_{1}$ and $n_{2}$, define the jump and average by

$$
\begin{array}{llrl}
{[v]=\left(\left.v\right|_{\kappa_{1}} n_{1}+\left.v\right|_{\kappa_{2}} n_{2}\right),} & \{v\} & =\frac{1}{2}\left(\left.v\right|_{\kappa_{1}}+\left.v\right|_{\kappa_{2}}\right), \\
{[\tau]=\left(\left.\tau\right|_{\kappa_{1}} \cdot n_{1}+\left.\tau\right|_{\kappa_{2}} \cdot n_{2}\right),} & \{\tau\} & =\frac{1}{2}\left(\left.\tau\right|_{\kappa_{1}}+\left.\tau\right|_{\kappa_{2}}\right) .
\end{array}
$$

On outer faces $F=\partial \kappa \cap \partial \Omega \in \mathcal{F}_{e}$, for some $\kappa \in \mathcal{K}$ with outer normal $n$, the jump and the average are defined as $[v]=\left.v\right|_{F} n$ and $\{v\}=\left.v\right|_{F}$ resp. $[\tau]=\left.\tau\right|_{F} \cdot n$ and $\{\tau\}=\left.\tau\right|_{F}$. Define the piecewise affine discontinuous finite element space by

$$
V_{h}^{1}=\left\{v_{h} \in L^{2}(\Omega):\left.v_{h}\right|_{\kappa} \in \mathbb{P}_{1}(\kappa), \forall \kappa \in \mathcal{K}\right\}
$$

and the enriched space by

$$
V_{b s}=V_{h}^{1} \oplus V^{b}, \text { with } V^{b}=\left\{v \in L^{2}(\Omega):\left.v(x)\right|_{\kappa}=\alpha_{\kappa} x \cdot x ; \text { where } \alpha_{\kappa} \in \mathbb{R}\right\},
$$

where $x=\left(x_{1}, \ldots, x_{d}\right)$ denotes the physical variables. We may then write the finite element formulation: Find $u_{h} \in V_{b s}$ such that

$$
a_{s}\left(u_{h}, v_{h}\right)=\left(f, v_{h}\right)_{\mathcal{K}} \quad \forall v_{h} \in V_{b s},
$$

where

$$
a_{s}\left(u_{h}, v_{h}\right)=\left(\nabla u_{h}, \nabla v_{h}\right)_{\mathcal{K}}-\left(\left\{\nabla u_{h}\right\},\left[v_{h}\right]\right)_{\mathcal{F}}-s\left(\left\{\nabla v_{h}\right\},\left[u_{h}\right]\right)_{\mathcal{F}},
$$

for $s \in\{-1,1\}$. This formulation is consistent and in the case $s=1$ also adjoint consistent. For the analysis of this method we introduce the triple norm

$$
\left\|v_{h}\right\|^{2}=\left\|v_{h}\right\|_{1, \mathcal{K}}^{2}+\left\|\tilde{h}_{\mathcal{F}}^{-\frac{1}{2}}\left[v_{h}\right]\right\|_{\mathcal{F}}^{2} .
$$


2.1. Properties of the enriched space $V_{b s}$. The motivation for the particular form of the enriched space is given in the following Lemma. The key idea is that the gradient of a function in $V_{b s}$ restricted to an element is in fact locally in the RaviartThomas space. Let $R T_{0}$ denote the space of Raviart-Thomas elements of order zero.

Lemma 2.1. For all $w_{h} \in V_{b s}$ there holds that $\left.\nabla w_{h}\right|_{\kappa} \in R T_{0}(\kappa)$, and for all $r_{h} \in R T_{0}(\kappa)$ there exists $w_{h} \in V_{b s}$ such that $\left.\nabla w_{h}\right|_{\kappa}=r_{h}$, for all $\kappa \in \mathcal{K}$.

Proof. See [6]. $\quad \square$ In particular this implies that $\left.\nabla u_{h}\right|_{\kappa} \cdot n_{F}$ is constant on each face $F \in \kappa$.

2.1.1. Projection. The key result for the stability of the method is the existence of we the following projection. We let $\left.\bar{v}\right|_{F}$ denote the average of the function $v$ over the face $F$.

Lemma 2.2 (Interpolant). Let $u_{h} \in V_{b s}$ be a fixed function. Then there exists a unique $w_{h} \in V_{b s}$ such that

$$
\begin{aligned}
\int_{\kappa} w_{h} d x & =0 \quad \forall \kappa \in \mathcal{K} \\
\left.\left\{\nabla w_{h}\right\}\right|_{F} \cdot n_{F} & =h_{F}^{-1} \overline{\left[u_{h}\right]} \cdot n_{F} \quad \forall F \in \mathcal{F} \\
\int_{F}\left\{w_{h}\right\} & =0 \quad \forall F \in \mathcal{F}_{i} .
\end{aligned}
$$

In addition the following a priori estimates holds

$$
\left\|\nabla w_{h}\right\|_{\mathcal{K}} \leq c\left\|\tilde{h}_{\mathcal{F}}^{-\frac{1}{2}} \overline{\left[u_{h}\right]}\right\|_{\mathcal{F}} \text { and }\left\|w_{h}\right\||\leq c|\left\|u_{h}\right\| \| .
$$

Proof. Let us first observe that the number of conditions on the projection is equal to the number of unknowns. The dimension of the finite element space $V_{b s}$ is $(d+2) N_{e l}$ where $N_{e l}$ denotes the number of elements in the mesh. On the other hand condition (4) enforces $N_{e l}$ constraints whereas conditions (5) and (6) demand $N_{F}+N_{F_{i}}$ constraints where $N_{F}$ and $N_{F_{i}}$ denote the number of the number of faces resp. the number of interior faces of the mesh. Observing that $N_{F}+N_{F_{i}}=(d+1) N_{e l}$ implies directly a square linear system to determine the projection. Since $v_{h}$ has zero mean over each element it satisfies the following strong Poincaré inequality

$$
\left\|w_{h}\right\|_{\mathcal{K}} \leq c\left\|\tilde{h} \nabla w_{h}\right\|_{\mathcal{K}}
$$

Now, we prove that any solution to the linear system (4)-(6) satisfies the a priori error estimate (7). Integrating by parts and using the properties of $w_{h}$, it follows that

$$
\begin{aligned}
\left\|\nabla w_{h}\right\|_{\mathcal{K}}^{2} & =-\underbrace{\left(\Delta w_{h}, w_{h}\right)_{\mathcal{K}}}_{=0}+\left(\left\{\nabla w_{h}\right\},\left[w_{h}\right]\right)_{\mathcal{F}}+\underbrace{\left(\left[\nabla w_{h}\right],\left\{w_{h}\right\}\right)_{\mathcal{F}_{i}}}_{=0} \\
& =\left(\tilde{h}_{\mathcal{F}}^{-1} \overline{\left[u_{h}\right]},\left[w_{h}\right]\right)_{\mathcal{F}} \leq\left\|\tilde{h}_{\mathcal{F}}^{-\frac{1}{2}} \overline{\left[u_{h}\right]}\right\|_{\mathcal{F}}\left\|\tilde{h}_{\mathcal{F}}^{-\frac{1}{2}}\left[w_{h}\right]\right\|_{\mathcal{F}} \leq c\left\|\tilde{h}_{\mathcal{F}}^{-\frac{1}{2}} \overline{\left[u_{h}\right]}\right\|_{\mathcal{F}}\left\|\nabla w_{h}\right\|_{\mathcal{K}}
\end{aligned}
$$

since $\left\{\nabla w_{h}\right\}$ and $\left[\nabla w_{h}\right]$ are constant along each face (see Lemma 2.1) and using a trace inequality followed by (8). The estimate (7) now is an immediate consequence of $(8)$. 
Since the linear system is square, existence and uniqueness of a solution of the linear system are equivalent. Let us denote by $A w=b$ the square linear system and assume that there is a vector $w_{1}$ and $w_{2}$ such that $A w_{i}=b, i=1,2$. Further let us denote the difference between them by $e=w_{1}-w_{2}$ and therefore $A e=0$. The a priori estimate (7) implies that $e=0$ and thus the solution is unique and hence the matrix is regular.

2.2. Stability and convergence of the bubble enriched DG-method. The stability of the bubble enriched DG-method is obtained by the satisfaction of the following inf-sup condition:

Theorem 2.3. (Discrete inf-sup condition) There exists a constant $c>0$ independent of $h$ such that for all $u_{h} \in V_{b s}$ there holds

$$
c \mid\left\|u_{h}\right\| \leq \sup _{v_{h} \in V_{b s}} \frac{a_{s}\left(u_{h}, v_{h}\right)}{\left\|v_{h}\right\| \mid}, \quad \text { for } s \in\{-1,1\} .
$$

Proof. First we take $v_{h}=u_{h}$ in a standard fashion and use that $c\left\|h_{\mathcal{F}}^{-\frac{1}{2}}\left[u_{h}\right]\right\|_{\mathcal{F}}^{2} \leq$ $\left\|h_{\mathcal{F}}^{-\frac{1}{2}} \overline{\left[u_{h}\right]}\right\|_{\mathcal{F}}^{2}+\left\|\nabla u_{h}\right\|_{\mathcal{K}}^{2}$.

$a_{s}\left(u_{h}, u_{h}\right)=\left\|\nabla u_{h}\right\|_{\mathcal{K}}^{2}-(1+s)\left(\left\{\nabla u_{h}\right\},\left[u_{h}\right]\right)_{\mathcal{F}} \geq \frac{1}{2}\left\|\nabla u_{h}\right\|_{\mathcal{K}}^{2}-c_{u}(1+s)^{2}\left\|\tilde{h}_{\mathcal{F}}^{-\frac{1}{2}} \overline{\left[u_{h}\right]}\right\|_{\mathcal{F}}^{2}$.

Second let $w_{h}$ denote the projection of Lemma 2.2, it follows that

$a_{s}\left(u_{h}, w_{h}\right)=-\left(\Delta u_{h}, w_{h}\right)_{\mathcal{K}}+\left(\left[\nabla u_{h}\right],\left\{w_{h}\right\}\right)_{\mathcal{F}_{i}}-s\left(\left\{\nabla w_{h}\right\},\left[u_{h}\right]\right)_{\mathcal{F}}=-s\left\|\tilde{h}_{\mathcal{F}}^{-\frac{1}{2}} \overline{\left[u_{h}\right]}\right\|_{\mathcal{F}}^{2}$.

Combining the results (9) and (10) we may take $v_{h}=u_{h}-s\left(\frac{1}{2}+c_{u}(1+s)^{2}\right) w_{h}$ to obtain after an application of a Poincaré inequality for piecewise $H^{1}$-functions that $a_{s}\left(u_{h}, v_{h}\right) \geq \frac{1}{2}\|\| u_{h} \|^{2}$. We conclude by using (7) to show that $\left\|v_{h}\right\| \mid \leq c\|\| u_{h}\|\|$.

Optimal convergence now follows in a standard fashion using the discrete inf-sup condition of Theorem 2.3, Galerkin orthogonality and approximation.

Theorem 2.4. Let $u \in H^{2}(\Omega)$ be the solution of (1) and $u_{h}$ the solution of $(2)$

$$
\left\|u-u_{h}\right\| \leq c h\|u\|_{2, \mathcal{K}} \quad \text { and if } s=1 \quad\left\|u-u_{h}\right\|_{0, \Omega} \leq c h^{2}\|u\|_{2, \mathcal{K}} .
$$

\section{REFERENCES}

[1] D. N. Arnold. An interior penalty finite element method with discontinuous elements. SIAM J. Numer. Anal., 19(4):742-760, 1982.

[2] D. N. Arnold, F. Brezzi, B. Cockburn, and L. D. Marini. Unified analysis of discontinuous Galerkin methods for elliptic problems. SIAM J. Numer. Anal., 39(5):1749-1779 (electronic), 2001/02.

[3] G. Baker. Finite element methods for elliptic equations using nonconforming elements. Math. Comp., 31:44-59, 1977.

[4] F. Brezzi and L. D. Marini. Bubble stabilization of discontinuous Galerkin methods. In W. Fitzgibbon, R. Hoppe, J. Periaux, O. Pironneau, and Y. Vassilevski, editors, Advances in numerical mathematics, Proc. International Conference on the occasion of the 60th birthday of Y.A. Kuznetsov, pages 25-36. Institute of Numerical Mathematics of The Russian Academy of Sciences, Moscow, 2006.

[5] E. Burman, A. Ern, I. Mozolevski, and B. Stamm. The symmetric discontinuous Galerkin method does not need stabilization in $1 \mathrm{~d}$ for polynomial orders $p \geq 2$. C. R. Acad. Sci. Paris, Ser. I, 345(10):599-6032, 2007. 
[6] E. Burman and B. Stamm. Low order discontinuous Galerkin methods for second order elliptic problems. Technical Report 04-2007, EPFL-IACS, 2007.

[7] M. G. Larson and A. J. Niklasson. Analysis of a family of discontinuous Galerkin methods for elliptic problems: the one dimensional case. Numer. Math., 99:113-130, 2004.

[8] M. G. Larson and A. J. Niklasson. Analysis of a nonsymmetric discontinuous Galerkin method for elliptic problems: stability and energy error estimates. SIAM J. Numer. Anal., 42(1):252264,2004

[9] J. T. Oden, I. Babuška, and C. Baumann. A discontinuous $h p$ finite element method for diffusion problems. J. Comput. Phys., 146(2):491-519, 1998.

[10] B. Rivière, M. F. Wheeler, and V. Girault. A priori error estimates for finite element methods based on discontinuous approximation spaces for elliptic problems. SIAM J. Numer. Anal., 39(3):902-931 (electronic), 2001.

E. Burman, Department of Mathematics, Mantell Building, University of Sussex, Falmer, Brighton, BN1 9RF, United Kingdom

E-mail address: Erik.Burman@epfl.ch

B. Stamm, iaCS/CMCS, station 8, École polytechnique fédérale de Lausanne, CH 1015, Lausanne, SWitzerLand

E-mail address: Benjamin.Stamm@epfl.ch 